\title{
Characteristics associated with HIV and HSV-2 among adolescents in Malawi
}

\author{
Barbara Mensch \\ Population Council \\ Erica Soler-Hampejsek
}

Follow this and additional works at: https://knowledgecommons.popcouncil.org/departments_sbsr-hiv

Part of the Demography, Population, and Ecology Commons, Family, Life Course, and Society Commons, International Public Health Commons, Medicine and Health Commons, and the Women's Health Commons How does access to this work benefit you? Let us know!

\section{Recommended Citation}

Mensch, Barbara and Erica Soler-Hampejsek. 2017. "Characteristics associated with HIV and HSV-2 among adolescents in Malawi," Project SOAR Results Brief. Washington, DC: Population Council. 


\section{Characteristics Associated with HIV and HSV-2 among Adolescents in Malawi}

The DREAMS (Determined, Resilient, Empowered AIDS-free, Mentored, and Safe) partnership aims to significantly reduce new HIV infections in adolescent girls and young women (AGYW) in Malawi and other sub-Saharan African countries. Through local and international partners, DREAMS is delivering a package of evidence-based interventions that go beyond the health sector, addressing the structural drivers that fuel AGYW's HIV risk. However, in order to have the greatest impact on the HIV epidemic, DREAMS needs to reach the most-at risk AGYW.

To better understand the epidemiological context for the DREAMS programming, Project SOAR analyzed previously collected quantitative cohort data from the Population Council's Malawi Schooling and Adolescent study (MSAS) ${ }^{1}$ to identify the factors associated with prevalence and incidence of HIV and HSV-2 (herpes simplex virus, commonly referred to as genital herpes). SOAR's analysis provides a deeper understanding of the epidemiological and gendered context of the HIV epidemic in Malawi.

\section{WHAT IS THE MSAS?}

MSAS is a six-round longitudinal survey of 2,649 in school $(1,764)$ and out of school $(885)$ female and male adolescents residing in Machinga and Balaka districts, two adjacent rural districts in the country's southern region where HIV prevalence is the highest in the country. MSAS began in 2007 when respondents were aged 14-17. The study re-interviewed the original sample at one to two year intervals from 2008 to 2013 ; the follow-up rate

\footnotetext{
${ }^{1}$ Data reported in this brief was originally collected with support by grants from the Eunice Kennedy Shriver National Institute of Child Health and Human Development (R01HD047764 and R01HD062155), and the Spencer Foundation. The content is solely the responsibility of the authors and does not necessarily represent the official views of the funding institutions. For more information about MSAS, please visit popcouncil.org/research/malawi-schooling-andadolescent-study.
}

\section{Understanding the changing HIV epidemic among young people}

- Examining prevalence and incidence data together can provide information on both the burden of the epidemic and the risk of infection in a certain population.

- Most studies examining HIV infection among young people in sub-Saharan Africa are based on cross-sectional data, and are therefore unable to assess HIV incidence.

- The youth cohort data from the MSAS provides accurate estimates of HIV prevalence over time and HIV incidence, thereby filling important data gaps.

- HSV-2, which is almost always sexually transmitted, is considered a reliable marker of sexual behavior among youth, who often underreport sexual activity.

- HSV-2 co-infection increases the risk of HIV transmission to uninfected partners.

ranged from 82 percent to 91 percent of the original baseline. The MSAS survey includes questions on household and family characteristics, educational attainment, schooling history and experiences, household labor and employment, health, marriage, and sexual behavior. Respondents aged 17-20 were tested for HIV and HSV-2 ${ }^{2}$ in Round 4 (2010), and Round 6 (2013), and were given their results if wanted and provided with counseling and referral if HIV positive.

${ }^{2}$ Blood specimens were collected via finger pricks. A serial algorithm was used for HIV rapid testing and positive samples were retested for status confirmation. The HSV-2 samples were tested using the Kalon ELISA HSV-2 antibody test. 


\section{ANALYSIS}

Prevalence of HIV and HSV-2 was computed at Round 6 in 2013 using testing data from all rounds. In order to identify which characteristics put young people at risk, multivariate logistic regression models were estimated to identify the most important characteristics associated with prevalence of infection when all other variables are controlled. Baseline characteristics considered included: age, ethnic group, district of residence, whether moved prior to 2007, orphanhood status, parental education, school enrollment, ability to read in English and Chichewa and numeracy score, sexually active, marital status, household assets, participation in sports and social clubs, number of male and female friends, experience of violence in the home, pregnancy experience or partner's pregnancy experience as well as baseline community characteristics (proportion of fathers who have completed primary school and household assets). For prevalence models, individual characteristics were measured in 2007 whereas HIV and HSV-2 status were measured in 2013; thus, the characteristics associated with HIV prevalence were measured, for the most part, prior to infection.
Incidence of HIV and HSV-2 between Rounds 4 and 6 (2010-2013) for those negative at Round 4 was also calculated. Bivariate Cox hazard models were used to estimate characteristics that significantly increased the risk of acquiring HIV or HSV-2 between 2010 and 2013 among those uninfected 2010. However, due to the low rates of HIV infection, our analysis may have had less power to identify all significant correlates of infection. Characteristics that may have changed since 2007 were measured as of Round 4 in 2010.

\section{RESULTS}

\section{HIV and HSV-2 prevalence}

As is true throughout the region, young women are much more likely to be infected; 7.0 percent of females tested positive for HIV compared with 1.7 percent of males. Prevalence of HSV-2 is quite high; as 24.3 percent of males and 33.6 percent of females were infected by Round 6 when aged 20-23 years.

\section{Adolescent men's and women's characteristics at baseline (2007)}

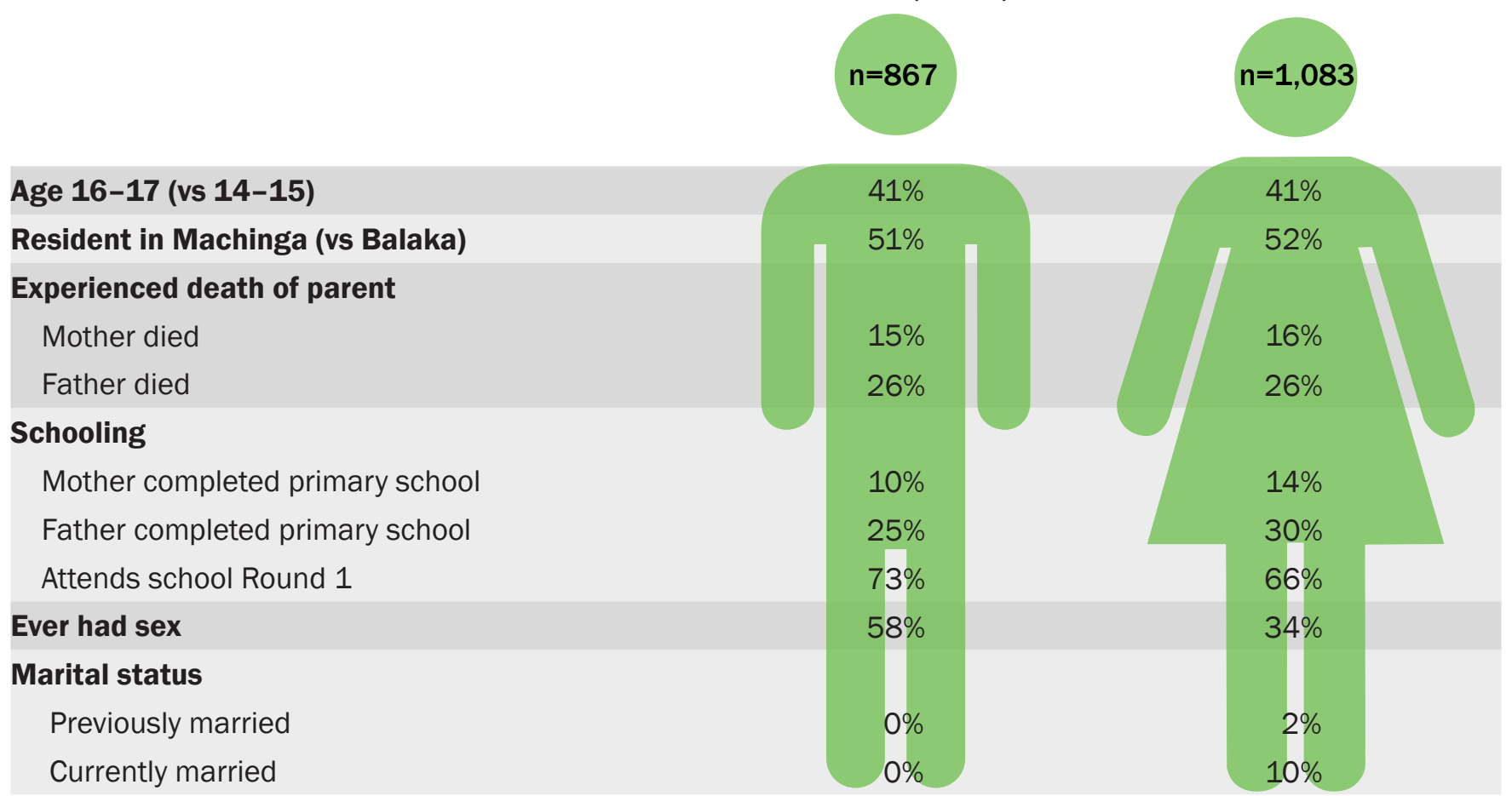


Characteristics associated with prevalence of HIV and HSV-2: Multivariate models

Characteristics associated with HIV
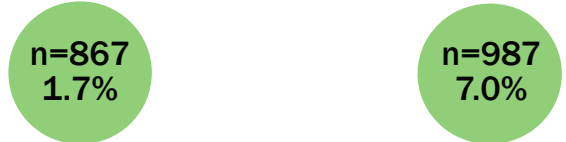

- Father has completed primary schooling

- Had male friends during childhood

- Previously married

- Currently married
- Currently working

- Mother died during childhood

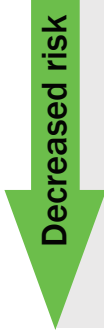

Characteristics associated with HSV-2

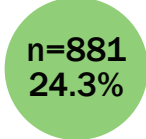

- Member of Yao ethnic group (vs. other)

- Participated in sports activities

- Community where most fathers had completed primary school

(None significant)
- Member of Yao ethic group (vs. other)

- Previously married $33.6 \%$

- Continued residence in same area

- Count of household assets

- Attended school during childhood

\section{HSV-2 and HIV incidence}

For HIV, the incidence rate was 3.4 per 1,000 person years among males and 9.9 per 1,000 person years among females. The estimated incidence rate for HSV-2 between 2010 and 2013 was 38.0 per 1,000 person years among males and 51.4 per 1,000 person years among females.

Characteristics associated with HIV and HSV-2 acquisition among adolescent males and females: Bivariate models

HIV acquisition (Incidence rate per 1,000)
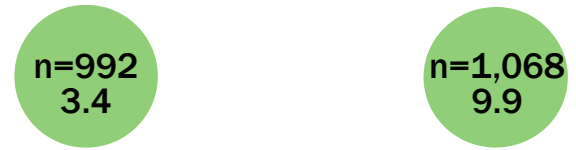

- Higher mean household assets childhood

- Father has completed primary school

(None significant)

\section{- Mother died during}




\section{KEY FINDINGS}

- Education is protective against HSV-2 infection for adolescent females; motivation to stay in school could be a deterrent to engaging in unprotected sexual activity.

- Early childbearing heightens HSV-2 risk for adolescent females, This may be a result of unprotected sexual activity with a main or marital partner.

- Parental death can heighten HIV risk for adolescent males, perhaps due to limited connectedness to the home or loss of social and financial support.

- Adolescent males and females residing in relatively better off areas as measured by parental education and household assets, are more likely to be infected with HIV and HSV-2. These areas are likely more densely settled, closer to paved roads and thus, have a more mobile population.

- Living in a high-risk district increases HIV and HSV-2 risk, as there is a higher likelihood that current or potential partners might already have one of these STIs thereby heightening the risk of acquiring HIV or HSV-2.

\section{RECOMMENDATIONS}

- Invest in education to reduce risk among adolescent females-with an emphasis on staying in school, enhancing the skills gained at school, delaying early marriage and pregnancy.

- Enhance HIV testing, early diagnosis, rapid link to treatment, and support for treatment adherence for adults to reduce orphanhood.

- Develop HIV prevention messages targeted toward mobile and adolescents residing in densely settled areas.

- Strengthen STI prevention and treatment programs in geographic areas of high risk.

- Sustain HIV prevention and treatment efforts in high-risk geographic areas to change the course of the epidemic in those areas.

For more information, please contact Barbara Mensch (bmensch@popcouncil.org), Nanlesta Pilgrim (npilgrim@ popcouncil.org), Sanyukta Mathur (smathur@popcouncil.org), or Lyson Tenthani (Itenthani@popcouncil.org).

Suggested citation: Mensch, Barbara S., and Erica Soler-Hampejsek. 2017. "Characteristics associated with HIV and HSV-2 among adolescents in Malawi," Project SOAR Results Brief. Washington, DC: Population Council. agreement funded by the President's Emergency Plan for AIDS Relief and the U. S. Agency for International Development (Agreement No. AIDOAA-A-14-00060). SOAR is able to accept funding from all USAID accounts.
Project SOAR/Population Council

4301 Connecticut Avenue, NW, Suite 280

Washington, DC 20008

Tel: +1 2022379400

e-mail: ProjectSOAR@popcouncil.org

popcouncil.org/ProjectSOAR

(๑)Population Council, October 2017 